\title{
Tolerance to lipopolysaccharide promotes an enhanced neutrophil extracellular traps formation leading to a more efficient bacterial clearance in mice
}

V. I. Landoni, ${ }^{\star}$ P. Chiarella, ${ }^{\star}$

D. Martire-Greco, ${ }^{\star}$ P. Schierloh, ${ }^{\star}$

N. van-Rooijen, ${ }^{\dagger}$ B. Rearte, ${ }^{*}$

M. S. Palermo, ${ }^{\star}$ M. A. Isturiz ${ }^{\star}$ and

G. C. Fernández*

${ }^{*}$ Immunology Department of the Instituto de Medicina Experimental (IMEX, CONICET) and Academia Nacional de Medicina de Buenos Aires, Argentina, and ${ }^{\dagger}$ Department of Molecular Cell Biology, Vrije University Medical Center, Amsterdam, the Netherlands

Accepted for publication 21 December 2011 Correspondence: V. I. Landoni, Immunology Department, Instituto de Medicina Experimental (IMEX, CONICET), Academia Nacional de Medicina, Pacheco de Melo 3081, C1425AUM, Buenos Aires, Argentina.

E-mail: vilandoni@yahoo.com.ar

\begin{abstract}
Summary
Tolerance to lipopolysaccharide (LPS) constitutes a stress adaptation, in which a primary contact with LPS results in a minimal response when a second exposure with the same stimulus occurs. However, active important defence mechanisms are mounted during the tolerant state. Our aim was to assess the contribution of polymorphonuclear neutrophils (PMN) in the clearance of bacterial infection in a mouse model of tolerance to LPS. After tolerance was developed, we investigated in vivo different mechanisms of bacterial clearance. The elimination of a locally induced polymicrobial challenge was more efficient in tolerant mice both in the presence or absence of local macrophages. This was related to a higher number of PMN migrating to the infectious site as a result of an increased number of PMN from the marginal pool with higher chemotactic capacity, not because of differences in their phagocytic activity or reactive species production. In vivo, neutrophils extracellular trap (NET) destruction by nuclease treatment abolished the observed increased clearance in tolerant but not in control mice. In line with this finding, in vitro NETs formation was higher in PMN from tolerant animals. These results indicate that the higher chemotactic response from an increased PMN marginal pool and the NETs enhanced forming capacity are the main mechanisms mediating bacterial clearance in tolerant mice. To sum up, far from being a lack of response, tolerance to LPS causes PMN priming effects which favour distant and local anti-infectious responses.
\end{abstract}

Keywords: clearance, in vivo, LPS, NETs, neutrophil

\section{Introduction}

Lipopolysaccharide (LPS) is the main component of the cell membrane of Gram-negative microorganisms and is an important cause of the sepsis syndrome [1,2]. LPS is a potent stimulator of the release of proinflammatory cytokines associated with septic shock. In experimental models, the inoculation of high doses of LPS induces pathological symptoms similar to those observed in the inflammatory phase of septic shock. However, exposure to repetitive low concentrations of LPS generates a refractory state towards its effects, known as tolerance to LPS [3]. Interestingly, tolerant cells or whole organisms can withstand a challenge of LPS with a concentration many times above that necessary to stimulate cells or to cause death. Tolerance has been postulated as a protective mechanism limiting excessive inflammation and preventing septic shock. However, the physiological significance and clinical relevance of LPS tolerance, especially in the context of a live bacterial infection, remain in question $[4,5]$. In particular, the relevance of polymorphonuclear neutrophils (PMN) in bacterial clearance is not understood fully in the tolerant context. Some studies reported an increased clearance of both Gram-positive and -negative organisms after tolerance induction [6-8]. Nevertheless, it is important to note that in these studies, bacteria were injected at the same site of the previous LPS administration, leading to an increased local influx of leucocytes prior to bacterial challenge. Therefore, in this study we used two different anatomical sites for tolerance induction (systemic) and bacterial challenge (peritoneal). In order to elucidate the role of PMN in bacterial clearance, local macrophages were depleted and focused our study on the analysis of different PMN bactericidal mechanisms involved in the process of bacterial clearance.

Neutrophils extracellular trap (NET) formation is a recently described anti-microbial mechanism of PMN which 
involves the release of chromatin decorated with granular proteins in order to bind extracellularly and kill microorganisms, respectively [9]. This prompted us to determine the contribution of this bactericidal mechanism in bacterial clearance in the tolerant state.

In this study, we found that tolerant animals clear a polymicrobial infection more efficiently than control animals. In this process, neither phagocytosis nor production of reactive species resulted in significant mechanisms to explain the mentioned difference. However, a higher PMN migration towards the infection site, presumably of PMN from an enhanced marginal pool, may account for this difference. Remarkably, LPS tolerance causes PMN priming effects that resulted in higher NET formation as the main mechanism involved in bacterial clearance.

\section{Materials and methods}

\section{LPS tolerance model}

$\mathrm{BALB} / \mathrm{c}$ mice were bred in the animal facility of the Department of Experimental Medicine, Academia Nacional de Medicina, Buenos Aires. Male mice aged 9-16 weeks and weighing 20-25 g were used throughout the experiments. They were maintained under a 12-h light-dark cycle at $22 \pm 2{ }^{\circ} \mathrm{C}$ and fed with standard diet and water ad libitum. The experiments performed were conducted according to principles set forth in the Guide for the Care and Use of Laboratory Animals [10]. A scheme of daily intravenous (i.v.) inoculation of low doses of LPS (Sigma, St Louis, MO, USA; O111:B4, with $0 \cdot 41 \%$ of protein content assay by the method of Lowry, as specified by the provider) was performed to render mice tolerant to LPS: days 1 and 2: $5 \mu \mathrm{g}$ per mouse and days 3 and 4: $10 \mu \mathrm{g}$ per mouse. Simultaneously, another group of mice was injected with the same volume of saline.

\section{Polymicrobial challenge}

The caecum of an untreated mouse was removed and the intestinal content was left at $37^{\circ} \mathrm{C}$ for $24 \mathrm{~h}$ in saline $10 \%$ fetal calf serum (FCS) in aerobic conditions; $5 \times 10^{7}$ aerobicenriched bacteria were injected intraperitoneally (i.p.) to control and tolerant mice. Routine bacteriological identification revealed that bacteria present in the inoculum belonged to Enterococcus and Streptococcus spp. Four hours later, blood was obtained and peritoneal lavage was performed with sterile saline solution as described below. Dosage of bacteria was performed in agar MacConkey plates.

\section{Depletion of peritoneal macrophages}

The depletion of peritoneal macrophages was performed by i.p. inoculation of liposomes loaded with clodronate, as described previously [11]. Liposomes loaded with clodronate were prepare as described previously $[12,13]$ and $0.7 \mathrm{mg}$ per mouse was injected.

\section{Blood sample collection and cell count determination}

Blood samples were obtained by puncture of the retroorbital plexus. A differential count of leucocytes in whole blood was performed with a Neubauer chamber using Turk's solution $(0.01 \%$ gentian violet in $3 \%$ glacial acetic acid) by light microscopy.

\section{Peritoneal lavage}

Animals were killed and the peritoneal content was collected by peritoneal lavage, as described previously [14]. Briefly, the skin of the abdomen was cut open in the midline after thorough disinfection and without injuring the muscle. Sterile saline solution $(2 \mathrm{ml})$ was injected into and aspirated out of the peritoneal cavity twice, using a sterile syringe and needle, to rinse out the content from the peritoneal cavity. Cells were washed and counted as described above for whole blood, and the peritoneal liquid was used for determination of bacterial cultures as described below.

\section{Bacterial cultures}

Blood and peritoneal lavage was obtained and cells were lysed with sterile distilled water. Aliquots of serial log dilutions of peritoneal fluid and blood were plated onto a selective growth medium for intestinal bacteria, MacConkey agar (Britania, Buenos Aires, Argentina). Plates were grown under aerobic conditions at $37^{\circ} \mathrm{C}$, and colonies were counted after overnight incubation. Bacterial counts are expressed as colony-forming units (CFU) per millilitre of peritoneal lavage or blood.

\section{In vivo phagocytosis of bacteria}

Fluorescein isothiocyanate (FITC)-Escherichia coli conjugation was performed as described previously [15]. FITC-E. coli $\left(1 \times 10^{7}\right)$ were injected i.p. After $4 \mathrm{~h}$, peritoneal lavage was performed and cells were incubated for 2 min with trypsin/ethylenediamine tetraacetic acid (EDTA) at $37^{\circ} \mathrm{C}$ (to release attached but not internalized bacteria). Immediately afterwards, 10\% FCS was added, and after washing, cells were incubated with phycoerythrin (PE)-conjugated Ly-6G for $30 \mathrm{~min}$ at room temperature. Cells were washed and resuspended in $0 \cdot 2 \mu \mathrm{l}$ of BD FACSflow (BD Bioscience, San Jose, CA, USA). The percentage of double-positive cells and the mean fluorescence intensity (MFI) of FITC were measured by flow cytometry.

\section{Contribution of reactive nitrogen and oxygen species}

Ten minutes before bacterial challenge, the inhibitor of nitric oxide synthase, $N \omega$-nitro-L-arginine methyl ester 
(L-NAME) (1.5 mg per mouse; Sigma), was inoculated i.p. After $4 \mathrm{~h}$, peritoneal lavage was performed and CFU were evaluated using MacConkey agar plates. NO production was determined by measurement of nitrite (in cell-free supernatants from peritoneal lavage) using the Griess reaction. Briefly, $50 \mu \mathrm{l}$ of sample aliquots were mixed with $50 \mu \mathrm{l}$ of Griess reagent (1\% sulphanilamide/0 $1 \%$ naphthylethylene diamine dihydrochloride/2\% phosphoric acid) in 96-well plates and incubated at $25^{\circ} \mathrm{C}$ for $10 \mathrm{~min}$. Absorbance at $540 \mathrm{~nm}$ was measured with a microplate reader. $\mathrm{NaNO}_{2}$ was used as standard to calculate nitrite concentrations. To determine the production of reactive oxygen species (ROS) on peritoneal migrated PMN, dihydrorhodamine (DHR)-123, a derivative of rhodamine 123 , was used. Briefly, PMN from the peritoneal lavage $\left(2 \times 10^{5}\right)$ were incubated $15 \mathrm{~min}$ at $37^{\circ} \mathrm{C}$ with $1 \mu \mathrm{M}$ DHR-123. Immediately afterwards, the green fluorescence was measured by flow cytometry.

\section{In vitro evaluation of neutrophil extracellular traps (NETs)}

A polymicrobial challenge was performed and $4 \mathrm{~h}$ later cells within the peritoneal cavity were collected. Stimulation of NETs formation was measured as described previously, with minor modifications [16]. Briefly, PMN $\left(0 \cdot 1 \times 10^{6}\right)$ were seeded gently onto glass coverslips coated with $0 \cdot 001 \%$ polylysine in a 48-well plate in triplicate, allowed to settle, and incubated in the presence of phorbol 12-myristate 13-acetate (PMA, $50 \mathrm{nM})$. In order to visualize incipient NETs and quantify accurately the PMN undergoing NETosis we chose an incubation period of $6 \mathrm{~h}$ at $37^{\circ} \mathrm{C} 5 \% \mathrm{CO}_{2}$. After the incubation period, samples were gently fixed with $4 \%$ paraformaldehyde (PFA), then washed with phosphate-buffered saline (PBS), and the percentage of NET-forming PMN were determined using epifluorescence microscopy by staining DNA with propidium iodide. At least 10 different fields were observed in each triplicate $(\times 400)$.

\section{In vivo evaluation of NETs formation}

In vivo, NET contribution was determined by i.p. inoculation of microccocal nuclease ( $\mu$ ccal, $100 \mathrm{U} /$ mouse, prepared in PBS $\times 1$ with $5 \mathrm{mM} \mathrm{Cl}_{2} \mathrm{Mg}$ and $5 \mathrm{mM} \mathrm{Cl}_{2} \mathrm{Ca}$; Worthington, Lakewood, NJ, USA) or $\mu$ ccal plus EDTA $(10 \mathrm{mM})$ in order to inactivate $\mu$ ccal activity, $10 \mathrm{~min}$ before i.p. bacterial challenge. After $4 \mathrm{~h}$, peritoneal cells were collected carefully and seeded gently on polylysine pretreated coverslides. After fixation with $2 \%$ PFA, samples were washed and NETs were stained with propidium iodide. Images for NETs evaluation were acquired using a FluoView FV1000 confocal microscope (Olympus, Tokyo, Japan) equipped with a Plapon $60 \times / 1.42$ objective lens and processed using Olympus FV10-ASW software by considering maximum fluorescence intensity projections along the whole Z-stack.

\section{Marginal pool cell collection}

Marginal pool PMN were obtained as described previously [17], with modifications. Under anaesthesia peripheral blood was taken from the retro-orbital plexus $(\approx 1 \mathrm{ml})$. Animals were then infused via the heart using a perfusion buffer consisting of PBS supplemented with 5 mM EDTA to avoid coagulation and $60 \mathrm{~g} / \mathrm{l}$ dextran (MW 500 000) to increase the colloidal osmotic pressure. Simultaneously, blood was drawn via the retro-orbital plexus. The first $3 \mathrm{ml}$ were discarded in order to ensure that the majority of the peripheral blood had been removed. A second sample was then obtained by further perfusion of $10 \mathrm{ml}$ of perfusion buffer and considered as the marginal pool. Peripheral blood and marginal pool cells were counted as described above. Cellular characterization of the marginal pool was performed by optical microscopy with May-GrünwaldGiemsa-stained smears.

\section{Peripheral blood PMN isolation}

Isolation of PMN from peripheral blood or marginal pool from individual mice was performed as described previously [18]. Briefly, PMN cells were obtained by centrifugation on a Ficoll-Hypaque density gradient $(\delta=1090$; Ficoll Pharmacia, Uppsala, Sweden; Hypaque; Wintthrop Products, Buenos Aires, Argentina) followed by sedimentation in 6\% dextran. Contaminating erythrocytes were removed by hypotonic lysis. After washing, the cells ( $>96 \%$ PMN on MayGrünwald-Giemsa-stained preparations) were resuspended in RPMI medium.

\section{Chemotaxis assay}

PMN chemotaxis was quantified using a modification of the Boyden chamber technique [19]. A cell suspension $(50 \mu \mathrm{l})$ containing $2 \times 10^{6}$ cells/ml in RPMI with $0 \cdot 5 \%$ bovine serum albumin (BSA) was placed into the top wells of a 48-well microchemotaxis chamber. A polyvinylpyrrolidone (PVP)-free polycarbonate membrane $(3-\mu \mathrm{m}$ pore size; Neuro Probe Inc., Gaithersburg, MD, USA) separated the cells from lower wells containing either RPMI or N-Formyl-Met-Leu-Phe (fMLP) $\left(10^{-7} \mathrm{M}\right)$. The chamber was incubated for $90 \mathrm{~min}$ at $37^{\circ} \mathrm{C}$ in a $5 \% \mathrm{CO}_{2}$ humidified atmosphere. After incubation, the filter was stained with Tincion-15 (Biopur SRL, Rosario, Argentina), and the number of PMN on the undersurface of the filter was counted in at least five random high-power fields (HPF) $\times 400$ for each of the triplicate filters.

\section{Statistical analysis}

Data are expressed as mean \pm standard error of individual mice. For two-group comparison, Student's $t$-test was 

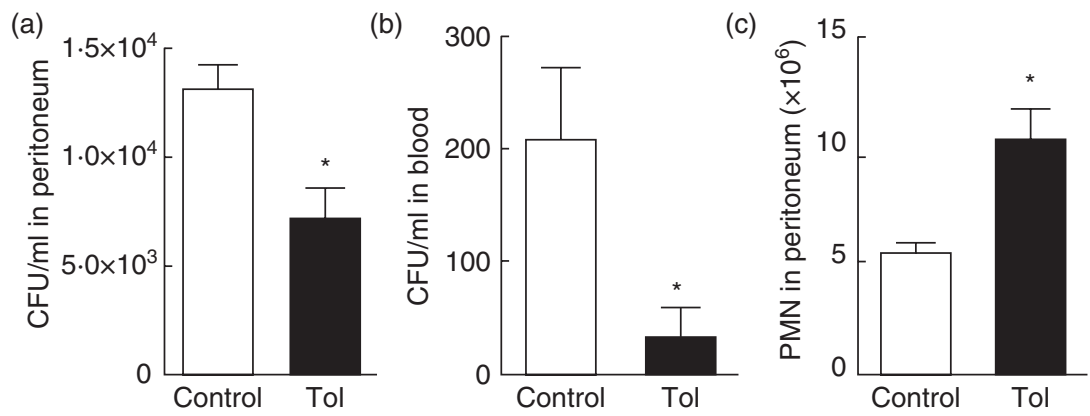

Fig. 1. Increased clearance of a polymicrobial challenge in the absence of macrophages in mice tolerant to lipopolysaccharide (LPS).

Colony-forming units (CFU) $\left(5 \times 10^{6}\right)$ of live intestinal bacteria were injected intraperitoneally (i.p.) after depletion of macrophages (by lip-clod treatment) in control mice and mice rendered tolerant to LPS by intravenous (i.v.) inoculation of LPS $(n=12)$. After 4 h, CFU were evaluated in peritoneal lavage (a) and blood (b). (c) The absolute number of total polymorphonuclear neutrophils (PMN) in the peritoneal lavage was determined by optical microscopy. ${ }^{\star} P<0.05$ versus control.

applied for normally distributed data (KolmogorovSmirnov normality test), except for non-parametric data from Figs $1 \mathrm{a}$ and $2 \mathrm{~b}$, where the Mann-Whitney $U$-test was applied. The comparisons between multiple groups were made by analysis of variance (ANOVA), applying Bonferroni's post test. $P<0.05$ was considered significant.

\section{Results}

Tolerant mice clear polymicrobial challenge more efficiently in the absence of macrophages

Mice were rendered tolerant by daily i.v. inoculation of low doses of LPS, and peritoneal macrophages were depleted by clodronate-loaded liposomes (lip-clod) treatment prior to an i.p. polymicrobial challenge. The depletion of macrophages within the peritoneal cavity was almost complete, as verified by staining of peritoneal cells with the specific F4/80 macrophage marker (25\% of total cells versus $0 \cdot 1 \%$ after lip-clod treatment). As depicted in Fig. 1, despite the absence of macrophages, $4 \mathrm{~h}$ after the inoculation of intestinal live bacteria the remaining CFU were lower in tolerant mice both in peritoneum (Fig. 1a) and blood (Fig. 1b) compared to control mice. Although circulating monocytes were not depleted by the lip-clod treatment, $4 \mathrm{~h}$ after the bacterial challenge few, if any, monocytes were observed within the peritoneal cavity.

This indicates that tolerant mice are more efficient in eliminating a bacterial challenge compared to control animals. More rapid clearance is unlikely, given that the number of CFU was still lower in tolerant mice at $24 \mathrm{~h}$ post-challenge (data not shown).

In parallel, we found an increase in the absolute number of PMN that have migrated to the infectious focus (Fig. 1c). No PMN were observed in the peritoneal cavity before bacterial challenge in control or tolerant mice (data not shown). The decreased remaining $\mathrm{CFU}$ in the peritoneum and the increased number of migrating PMN in tolerant mice were also observed in the absence of the lip-clod treatment $\left(\mathrm{CFU} \times 10^{4} / \mathrm{ml}\right.$ in peritoneum: control $=1 \cdot 7 \pm 0 \cdot 4$; tolerant $=0.4 \pm 0 \cdot 1^{*} ; \quad \mathrm{PMN}$ in peritoneum $\times 10^{6}$ : control $=1 \cdot 3 \pm 0 \cdot 2$, tolerant $=5 \cdot 2 \pm 1 \cdot 6^{\star}, n=6,{ }^{\star} P<0 \cdot 05$ versus
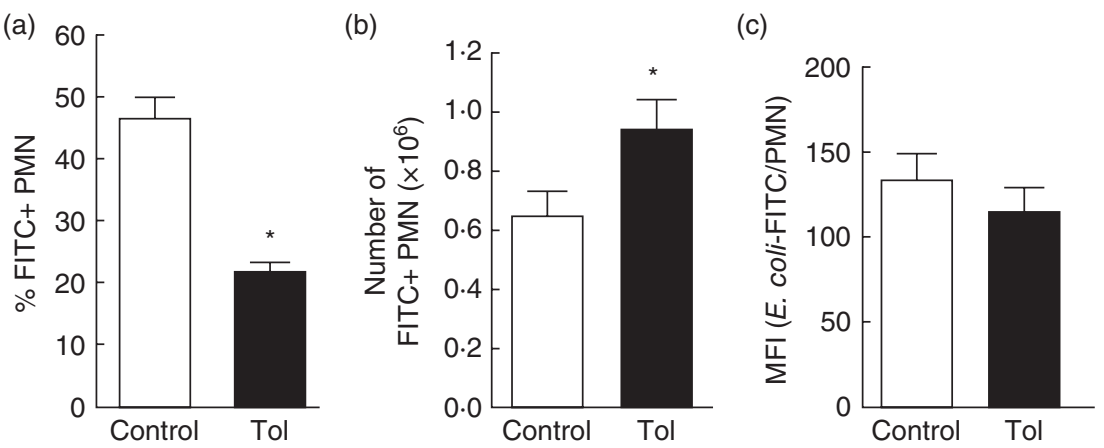

Fig. 2. Evaluation of in vivo phagocytosis of bacteria. Tolerance was developed by intravenous (i.v.) inoculation of lipopolysaccharide (LPS). Dead fluorescein isothiocyanate (FITC)-Escherichia coli was injected intraperitoneally (i.p.) in control and tolerant animals and 4 h later peritoneal lavage was performed $(n=12)$. The \% (a) and mean fluorescence intensity (MFI, c) of FITC + polymorphonuclear neutrophils (PMN) (identified as Ly- $6 \mathrm{G}^{+}$cells) were determined by flow cytometry. The absolute number of FITC + PMN (b) was calculated using the absolute number of PMN in the peritoneal lavage and the $\%$ of FITC + PMN shown in (a); ${ }^{\star} P<0.05$ versus control. 
control). Additionally, liposomes per se did not cause migration of PMN to the peritoneal cavity. These results reveal a key role of PMN in the resolution of local bacterial challenge, especially in tolerant mice.

\section{Tolerant mice have increased number of PMN which phagocyte bacteria}

In order to determine further the underlying mechanisms for the increased bacterial clearance observed in the tolerant state, the phagocytic ability of PMN was evaluated in situ by injecting death E. coli conjugated to FITC (FITC-E. coli) in the peritoneal cavity in macrophage-depleted mice. After $4 \mathrm{~h}$, peritoneal lavage was performed and FITC + PMN (Ly$6 \mathrm{G}^{+}$) were determined by fluorescence activated cell sorter (FACS) flow cytometry. As depicted in Fig. 2a, we found that the percentage of FITC + PMN was decreased in tolerant animals. However, as an increased absolute number of migrated PMN were found in tolerant compared to control mice (number of PMN $\left(\times 10^{6}\right)$ : control $=1 \cdot 5 \pm 0 \cdot 1$, tolerant $\left.=4 \cdot 6 \pm 0 \cdot 5^{\star},{ }^{\star} P<0 \cdot 05\right)$. When these absolute values were use to calculate the number of FITC + PMN, a statistically significant increase was observed in tolerant mice (Fig. 2b). No differences in the amount of FITC-E. coli per individual PMN (MFI) were observed between control and tolerant PMN (Fig. 2c).

These results indicate that although phagocytosis per se is not altered in the state of tolerance to LPS, there is a higher phagocytic index as a result of the increased migration of $\mathrm{PMN}$ at the site of bacterial challenge.

\section{Reactive species do not account for the enhanced bacterial clearance in tolerant mice}

In order to determine the contribution of nitrogen reactive species to the enhanced clearance, experiments were performed in macrophage-depleted mice by injecting a nitric oxide synthase (NOS) inhibitor, L-NAME, in the peritoneal cavity $10 \mathrm{~min}$ before bacterial challenge. After $4 \mathrm{~h}$, although remaining CFU were still statistically lower in the tolerant group, both control and tolerant mice showed an approximately fourfold increase in CFU when L-NAME was administered (Fig. 3). Within each group, the inhibition of NOS did not affect the migration of PMN to the site of bacterial inoculation (data not shown). This result indicates that NO production contributes to the bacterial clearance equally and, therefore, this mechanism cannot account for the more efficient clearance observed in tolerant animals.

Additionally, when hydrogen peroxide generation was evaluated in individual PMN using DHR by FACS in PMN that have migrated to the peritoneal cavity after the challenge, no differences were observed in the MFI of DHR in PMN from control and tolerant mice. As no PMN were present in the peritoneal cavity before bacterial challenge, PMN from peripheral blood were used to determine whether

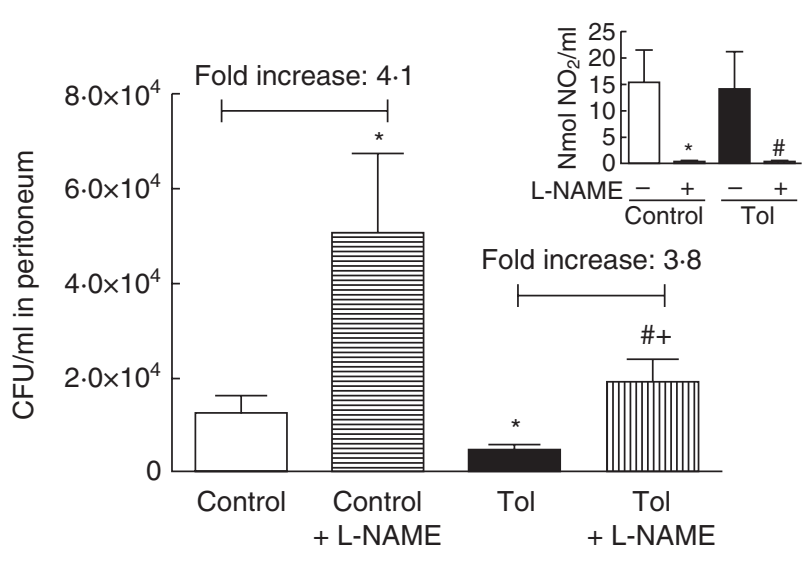

Fig. 3. The increased bacterial clearance in tolerant mice is not affected by inhibition of reactive nitrogen species. In vivo contribution of reactive nitrogen species production was determined by inoculating the inhibitor of reactive nitric oxide synthase, $N \omega$-nitro-L-arginine methyl ester (L-NAME) ( $1.5 \mathrm{mg}$ per mice) $10 \mathrm{~min}$ before bacterial challenge in control and tolerant mice. After $4 \mathrm{~h}$, colony-forming units (CFU) were evaluated using agar MacConkey in peritoneal lavage. The numbers at the top of the bars indicate the fold increase of $\mathrm{CFU} / \mathrm{ml}+\mathrm{L}-\mathrm{NAME}$ versus untreated mice. Inset: nmol of $\mathrm{NO}_{2} / \mathrm{ml}$ were determined in the experimental groups by the Griess method; $n=9,{ }^{\star} P<0.05$ versus control, $\# P<0.05$ versus tolerant, $+P<0.05$ versus control + L-NAME.

hydrogen peroxide generation in migrated PMN increased above that of unstimulated PMN. Values obtained for peripheral blood PMN before challenge were five times lower than those obtained for migrated PMN after bacterial challenge (MFI DHR from blood PMN before challenge: control $=23 \cdot 4 \pm 1 \cdot 87 ; n=10 ;$ tolerant $=21 \cdot 8 \pm 1 \cdot 3, n=12$; MFI DHR from peritoneal migrated PMN after challenge: control $=103 \cdot 5 \pm 8 \cdot 1 ; n=10$; tolerant $=99 \cdot 6 \pm 5 \cdot 2, n=12$ ).

Together, these results suggest that reactive species generation is not the major mechanism involved in the increased clearance mediated by PMN in the tolerant state.

\section{Neutrophil extracellular traps are involved in the enhanced clearance in tolerant mice}

In addition to the more traditional mechanism of phagocytosis to kill bacteria, activation of PMN causes the release of DNA structures (NET). We first evaluated the efficiency of migrated PMN to form NETs in vitro. PMN were collected $4 \mathrm{~h}$ after the bacterial challenge and NETs were induced by incubation with the potent protein kinase $\mathrm{C}$ (PKC) activator phorbol 12-myristate 13-acetate (PMA), as described previously [16]. After an additional $6 \mathrm{~h}$, the number of PMN undergoing NETosis was quantified microscopically. Interestingly, the percentage of NETforming PMN was higher in PMN from tolerant compared to control animals (Fig. 4a).

Moreover, NETs contribution in vivo in tolerant mice was measured by inoculating microccocal nuclease ( $\mu$ ccal, 
(a)

Fig. 4. Enhanced neutrophil extracellular traps (NETs) formation is involved in the increased bacterial clearance in tolerant mice. (a) In vitro NETs formation capacity was evaluated on polymorphonuclear neutrophils (PMN) recovered from the peritoneal cavity $4 \mathrm{~h}$ after polymicrobial challenge. PMN were seeded and treated with phorbol myristate acetate (PMA) for $6 \mathrm{~h}$. The percentage of PMN-forming NETs (NETotic PMN) was determined using epifluorescence microscopy by staining DNA with propidium iodide; $n=6,{ }^{\star} P<0.05$ versus control. (b) In vivo NET contribution was determined by inoculating micrococcal nuclease ( $\mu$ ccal) or $\mu c c a l+$ ethylenediamine tetraacetic acid (EDTA) 10 min before bacterial challenge in control and tolerant mice. Four hours later colony-forming units (CFU) were evaluated using agar MacConkey in peritoneal lavage; $n=13,{ }^{\star} P<0.05$ versus control, $\# P<0.05$ versus tolerant, $+P<0.05$ versus tolerant $+\mu$ ccal. (c-f) Four hours after the baterial challenge peritoneal, PMN were carefully collected and seeded onto coverslides. DNA was stained with propidium iodide and NETs were visualized by confocal microscopy $(\times 200$ and insets $\times 600)$. Asterisks indicate propidium iodide-stained bacteria. Representative microphotographies of an independent experiment are shown from one control (c), tolerant (d), tolerant with $\mu \mathrm{ccal}$ treatment (e) and tolerant with $\mu \mathrm{ccal}$ inactivated with EDTA (f).

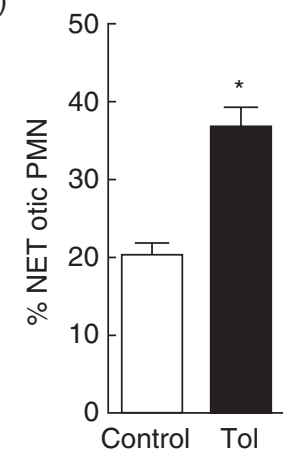

(b)
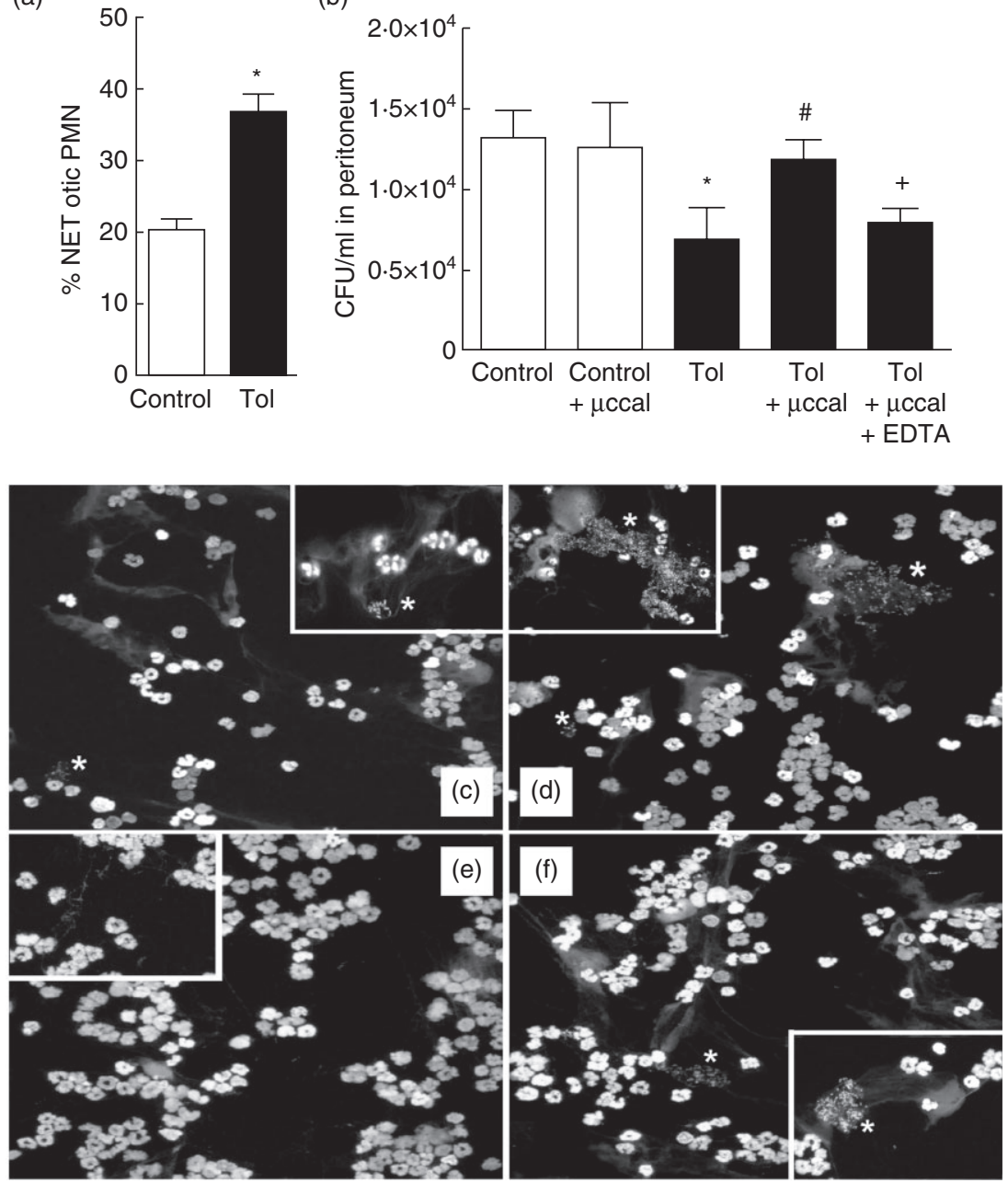

to digest chromatin) in the peritoneal cavity 10 min before bacterial challenge. As depicted in Fig. 4b, $\mu$ ccal treatment increased the CFU values in tolerant mice reaching control values, and this effect was reversed when $\mu$ ccal was inactivated by a 5-min preincubation with EDTA immediately before injection. Although NETs were visualized in PMN from control mice (Fig. 4c), CFU values were not affected by $\mu c c a l$ treatment. Within each group, $\mu$ ccal treatment did not modify the migration of PMN to the site of bacterial inoculation (data not shown).

In vivo results were also corroborated by confocal microscopy (Fig. 4c-f). Analysis of the microphotography obtained revealed some interesting aspects. On one hand, and confirming in vitro results, PMN from tolerant mice showed larger amounts of bacteria trapped in NETs (Fig. 4d). On the other hand, NETs integrity was clearly affected when $\mu$ ccal was employed (Fig. 4e) and this was reversed by EDTA preincubation (Fig. 4f).

Taken together, these results indicate clearly that NET formation is crucial for the increased clearance mediated by $\mathrm{PMN}$ in the tolerant state.
Increased PMN in the marginal pool compartment with higher migratory capacity in tolerant mice

As we have demonstrated previously, an increased number of PMN arriving to the site of bacterial challenge could be a critical determinant to the increased clearance observed in tolerant mice. It is well established that a considerable number of PMN are retained within the intravascular compartment, which is referred commonly as the marginal pool [20-22]. This marginal pool PMN is thought to be mobilized promptly immediately after a chemotactic signal. Therefore, in order to determine whether an increased marginal pool PMN could account for the increased number of migrated PMN after bacterial challenge, both the absolute number of PMN in peripheral blood and within the marginal pool after tolerance have been established. Figure 5 a shows that in spite of having similar numbers of PMN in circulation, PMN within the marginal pool were increased in tolerant animals. In agreement with Klonz et al. [17], the composition of the marginal pool was constituted by PMN and other cells such as lymphocytes and monocytes. Optical microscopy exami- 
(a)

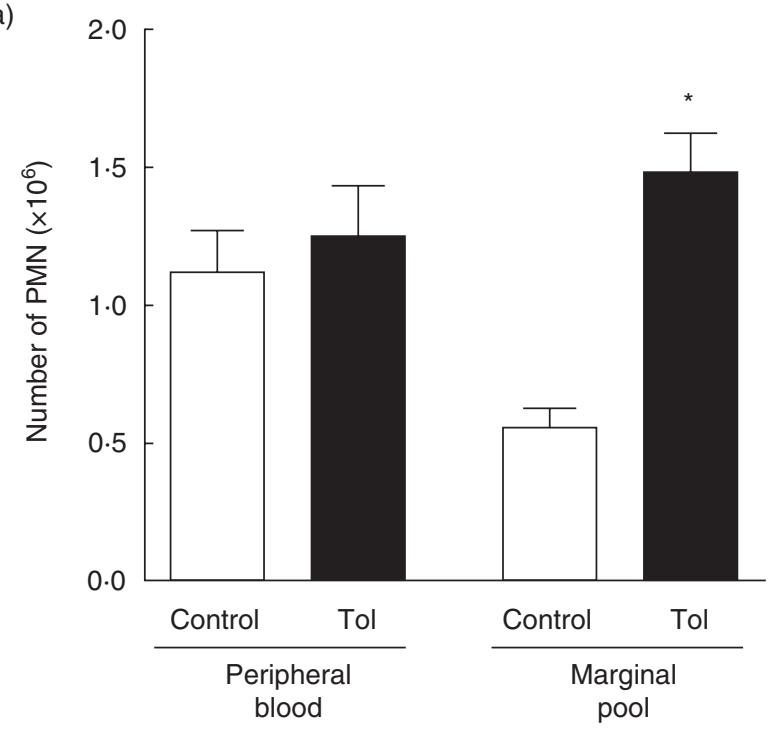

(b)

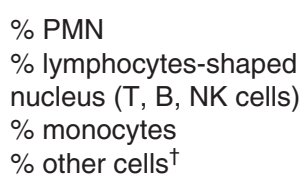

Control

Tol

$13 \cdot 8 \pm 1 \cdot 4 \quad 22 \cdot 4 \pm 2 \cdot 1^{*}$

$20 \cdot 3 \pm 5 \cdot 4 \quad 17 \cdot 9 \pm 1 \cdot 7$

$62 \cdot 5 \pm 7 \cdot 4 \quad 51 \cdot 3 \pm 1 \cdot 3$

$2 \cdot 8 \pm 2 \cdot 8 \quad 7 \cdot 9 \pm 1 \cdot 7$

(c)

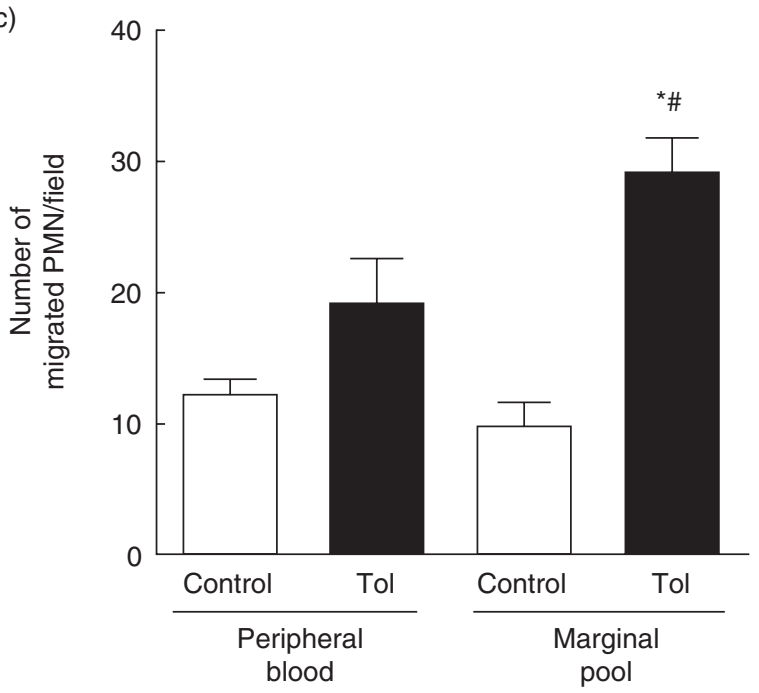

nation using May-Grümwald-Giemsa staining revealed a statistically significant increase in the percentage of PMN (Fig. 5b and c). Moreover, FACS analysis revealed similar percentages of double-positive Ly-6G (specific for PMN) CD11b cells (\% Ly-6G ${ }^{+}$CD $11 b^{+}$cells: control = 12.4 $\pm 0 \cdot 3$; tolerant $=25 \cdot 6 \pm 2 \cdot 1^{\star}, n=6,{ }^{\star} P<0 \cdot 05$ versus control). The expression of CD11b or Ly-6G within Ly- $6 \mathrm{G}^{+}$CD $11 b^{+}$cells was similar between marginal and blood PMN (data not shown).

Additionally, we tested the migratory capacity of PMN within these two compartments in vitro using fMLP, the most potent chemotactic factor present in all bacteria. We
Fig. 5. The marginal pool of polymorphonuclear neutrophils (PMN) is increased in tolerant mice. (a) The absolute number of PMN present in peripheral blood and marginal pool were determined as described in Materials and methods; $n=18,{ }^{\star} P<0.05$ versus control marginal pool. (b) Cellular composition of the marginal pool as determined by light microscopy using May-Grünwald-Giemsa staining; $n=6,{ }^{\star} P<0.05$ versus control. $\dagger$ Very large cells that could not be assigned to any category. (c) In vitro chemotactic migration of PMN against N-Formyl-Met-Leu-Phe (fMLP). Isolated PMN from peripheral blood and marginal pool $\left(1 \times 10^{5}\right)$ were allowed to migrate through a polycarbonate filter for $90 \mathrm{~min}$ at $37^{\circ} \mathrm{C}$ with $\mathrm{CO}_{2}$ in response to fMLP $\left(10^{-7} \mathrm{M}\right)$. Then, the filter was fixed and stained and the number of chemotactic cells was counted in five random high-power fields $(\times 400)$ for each of triplicate samples. The results were expressed as the number of migrated PMN per field; $n=6$ ${ }^{\star} P<0.05$ versus control marginal pool and peripheral blood, $\# P<0.05$ versus tolerant peripheral blood.

found that PMN from the marginal pool of tolerant mice showed the highest migratory capacity compared to both control marginal pool and tolerant peripheral PMN (Fig. 5c).

These results indicate that an increased PMN from the marginal pool with a higher migratory capacity could also contribute to the more efficient bacterial clearance in tolerant state.

\section{Discussion}

The means by which the host is able to combat bacterial infection within the peritoneal cavity has been of interest to investigators for more than a century. From the outset, it was clear that bacterial removal and phagocytosis were important host defences [23]. During the tolerant state, active important immune mechanisms are mounted. In this sense, some studies have reported an increased clearance of both Gram-positive and -negative organisms after induction of tolerance to LPS $[6,24,25]$. The goal in the present study was to define more precisely the role of PMN in host defence in tolerant mice and to determine the role of some of the most relevant PMN bactericidal components in bacterial clearance during experimental peritonitis. Our experiments were conducted using two different anatomical sites for LPS administration to induce tolerance and for bacterial challenge. As PMN are the first cells arriving at the site of infection, an advantage of this experimental setting is that, focusing on early phases of the induced peritonitis, it allows to study the relevance of the migration process of PMN itself. Additionally, different critical steps of PMN bactericidal mechanisms can also be evaluated by local administration of inhibitory factors, determining the precise impact of these mechanisms on bacterial clearance.

Although both PMN and macrophages are crucial for the resolution of an infection, the functional state of macrophages during tolerance to LPS has been studied 
widely both in vivo and in vitro [26-28]. However, the relevance of the bactericidal capacity of PMN in this context has been less explored. Therefore, in order to elucidate the actual contribution of PMN, by depleting peritoneal macrophages with clodronate-loaded liposomes, we created a system where the contribution of macrophages does not mask the results of the phenomena we want to study. We demonstrated that the clearance of a polymicrobial challenge was highly efficient in the tolerant state, even in the absence of macrophages. This could not be attributed to a higher phagocytic capacity of migrated PMN per se, or a higher leakage of bacteria to the peripheral blood. Our results are in line with the finding of Murphey et al., who found that the increased clearance of Gram-positive bacteria in tolerant mice was not related to differences in their phagocytic activity [29].

PMN migration to infectious foci is extremely important for the local control of bacterial growth and consequently for the prevention of bacterial dissemination. It has been shown that the failure of PMN to migrate to the infection site in lethal sepsis is accompanied by an increased number of bacteria in the peritoneal fluid and in the circulation and by a high mortality rate of the host [30,31]. We found an increased number of PMN that have migrated to the peritoneal cavity, the site of bacterial challenge. Enhanced PMN migration in the tolerant state was observed both in the presence or absence of macrophages, thus indicating that the signals secreted from resident activated peritoneal macrophages may not be determinant for attracting PMN in a context of high chemotactic bacterial factors. Additionally, the number of migrated PMN was higher when macrophages were depleted, even though the remaining bacteria (CFU/ml) were similar, suggesting compensatory migratory mechanisms in order to achieve a similar outcome. As it has been theorized that cells from the marginal pool are mobilized more readily in response to infections, the higher number of PMN within the marginal pool, together with the observed increased chemotactic activity towards fMLP in vitro of PMN from the marginal pool, may explain the increased number of cells at the infectious site after bacterial challenge in tolerant mice. Therefore, LPS priming leads not only to a redistribution of these PMN within the body, but also sets up mechanisms allowing a quick response when a danger signal arrives on the scene. Our results may contradict what has been described in severe septic patients. It has been shown that PMN from patients displayed a marked reduction in their chemotactic response compared with PMN from healthy subjects [32]. However, this discrepancy may be due to the fact that migration assays in patients were conducted using blood PMN, which may represent a different population than the one that first responded to the septic stimulus. Taking our results into account, it can be hypothesized that early LPS exposure causes priming or activation of PMN that become part of the marginal pool and are delivered rapidly to infected tissues, leaving less responsive (or deranged) cells in the peripheral circulation, accounting for two different populations of PMN with distinct responses. This phenomenon, known as compartmentalization, implies that the immune status of leucocytes within inflammatory foci displays a different status than the cells isolated from the bloodstream, and has been demonstrated in both experimental animal models and human volunteers for lung versus blood PMN. In this sense, Abraham et al. clearly demonstrated in murine models of endotoxaemia and haemorrhagic shock that lung-derived PMN displayed activation of transcriptional regulatory factors and increased expression of inflammatory cytokines, not observed in blood PMN [33,34]. Additionally, Coldren et al. reported that air space and circulating PMN showed a different gene expression pattern when human volunteers were exposed to LPS by bronchoscopic instillation [35]. In our study, a similar type of compartmentalization can be proposed between blood versus marginal pool PMN in tolerant mice. Studies comparing the expression of typical adhesion molecules or functional response in normal rats could not address any differences between blood versus marginal pool PMN $[17,36]$. However, it is possible that even though the functional capacities of cells from the circulating or marginated pools should be identical in normal conditions, activating/ priming stimuli (such as LPS or soluble factors generated in response to it) may trigger the mechanisms necessary to bring out differences between PMN from these two compartments, as observed here. To test this hypothesis, we are now exploring the expression of different chemotactic receptors and making a functional comparative study between marginal pool and blood PMN in tolerant mice.

The contribution of two other key microbicidal mechanisms was explored in this study: the generation of reactive species and NET formation. When nitrogen reactive species generation was blocked in vivo before bacterial challenge, both tolerant and control mice decreased bacterial clearance equally. These results reveal that tolerance to LPS failed to trigger any effect in priming this particular bactericidal mechanism and eventually, although still relevant to clear the infection, does not contribute to the enhanced clearance observed in tolerant mice. It is important to note that, in our conditions, the number of PMN arriving to the peritoneum was still augmented in tolerant mice after blockade of nitric oxide generation, suggesting that LPS effects on marginal pool PMN regarding migratory capacity may not be regulated by nitrogen-derived reactive species.

NETs play an important role in innate immunity to microbial infections. NETs have been described in several species, and their role in infection has not been addressed in vivo, partly because of the lack of optimal experimental models. In this sense, we develop a novel tool to investigate not only NET formation but also their relevance at the site of an active infection. We found that disruption of NETs by microccocal nuclease abolished the increased clearance observed in tolerant animals reaching the amounts of bac- 
teria found in control mice, without affecting directly the number of PMN that migrate to the peritoneum. Unexpectedly, the disruption of NETs in control mice did not modify bacterial clearance. In this regard, our in vitro results and those described by others [37] indicate that only a small percentage of murine PMN undergo NETosis after PMA stimulation (20-30\%). Therefore, under normal conditions the final outcome after abolishing NETs may not be affected. In this sense, it is possible that the impact of this mechanism in bacterial clearance would be similar to other microbicidal mechanisms (i.e. phagocytosis, ROS generation) and, therefore, the relevance of NETs becomes more evident in a primed (tolerant) context where the clearance of bacteria must be optimized. In line with this hypothesis, the NETs visualized from tolerant mice seemed to bind greater amounts of bacteria than NETs from control PMN, suggesting that NETs in tolerant PMN may also be more functional. In this sense, LPS priming may somehow favours the NETotic pathway over other mechanisms to eliminate an infection. In line with this, others have proposed that PMN undergo NETosis rather than the more classical phagocytosis to sieze bacteria under stressful conditions [38]. Therefore, tolerance to LPS can be considered a putative scenario where this kind of hierarchy of bactericidal mechanisms occurs. Moreover, the efficiency of PMN to undergo NETosis was also increased in the tolerant context, as revealed using PMA, indicating that not only a higher number of migrated PMN, leading to an increase number of NETs, but also intrinsic differences in the ability of individual PMN to form NETs, account for the increased bacterial clearance in tolerant mice. These results highlight the contribution of NETosis as a key active mechanism for in vivo resolution of infections in the tolerance to LPS state.

It has been described that NETs are dependent upon ROS generation after PMN activation [16,37]. Moreover, NET formation has been correlated with the amount of ROS produced [37]. In our study we cannot attribute the higher NET-forming capacity of PMN from tolerant mice to a concomitant higher generation of ROS, as the amount of $\mathrm{H}_{2} \mathrm{O}_{2}$ per peritoneal PMN from control and tolerant mice was similar. This reveals that, independently of the amount of ROS triggered, LPS tolerance may be favouring the formation of NETs by other mechanisms, which are currently being investigated. Considering that chemokines (and probably other unknown factors) can also induce NETs $[9,39]$, ongoing studies will address whether bacterial challenge triggers a higher local (peritoneal) chemotactic response in tolerant mice that can explain the observed higher induction of NETs.

In summary, the higher number of PMN arriving at the site of bacterial challenge, presumably coming from the marginal pool with an increased chemotactic response, together with an enhanced NET-forming ability of the migrated $\mathrm{PMN}$, as demonstrated in vitro, seem to be the main mechanisms accounting for a more efficient prevention of bacterial spreading in the state of tolerance. These interesting results open a wide field for elucidating the relevance of marginal pool PMN function in the context of infections and the regulating mechanism conferred by LPS priming. There is evidence that indicate that LPS tolerance can also cause immunosuppression in mice. Lu et al. have demonstrated that the inoculation of a pathogenic strain of E. coli causes $30 \%$ mortality in LPS-primed mice versus $0 \%$ in PBSprimed mice [40]. We have also observed a state of immunosuppression after LPS tolerance that involves only adaptive responses, such as $\mathrm{T}$ cell proliferation and antibody production [41]. Moreover, LPS-immunosuppressed mice survived a lethal dose of intestinal bacteria (three times higher than the one used in this manuscript) compares to control mice that died within $24 \mathrm{~h}$ (100\% survival in LPS versus $0 \%$ survival in control mice). In this sense, we believe that LPS priming of PMN mechanisms involved in the increased bacterial clearance in tolerant mice may be triggered in an attempt to compensate the deficiencies observed in the adaptive immune response. In line with this proposition, Parker et al. have shown that LPS induce a priming, rather than a tolerant effect, on human PMN [42].

Finally, as tolerance has been postulated as a protective mechanism limiting excessive inflammation and preventing septic shock, our results suggest that LPS exposure can induce PMN priming effects which may favour distant antiinfectious responses.

\section{Acknowledgements}

The authors thank Nora Galassi, Marta Felippo, Héctor Costa and Gabriela Camerano for their excellent technical assistance. We especially want to thank Juan Ignacio Basile for his assistance in migration studies. This work was supported by grants from Alberto J. Roemmers, Consejo Nacional de Investigaciones Científicas y Técnicas (CONICET) and Agencia Nacional de Promoción Científica y Tecnológica, Argentina.

\section{Disclosure}

The authors have no conflicts of interest.

\section{References}

1 Natanson C, Hoffman WD, Suffredini AF, Eichacker PQ, Danner RL. Selected treatment strategies for septic shock based on proposed mechanisms of pathogenesis. Ann Intern Med 1994; 120:771-83.

2 Vincent JL, Sakr Y, Sprung CL et al. Sepsis in European intensive care units: results of the SOAP study. Crit Care Med 2006; 34:34453.

3 Fan H, Cook JA. Molecular mechanisms of endotoxin tolerance. J Endotoxin Res 2004; 10:71-84.

4 Van Epps HL. Ignoring endotoxin. J Exp Med 2006; 203:1137. 
5 Cavaillon JM, Adib-Conquy M. Bench-to-bedside review: endotoxin tolerance as a model of leukocyte reprogramming in sepsis. Crit Care 2006; 10:233.

6 Varma TK, Durham M, Murphey ED et al. Endotoxin priming improves clearance of Pseudomonas aeruginosa in wild-type and interleukin-10 knockout mice. Infect Immun 2005; 73:7340-7.

7 Lehner MD, Ittner J, Bundschuh DS, van Rooijen N, Wendel A, Hartung T. Improved innate immunity of endotoxin-tolerant mice increases resistance to Salmonella enterica serovar typhimurium infection despite attenuated cytokine response. Infect Immun 2001; 69:463-71.

8 Wheeler DS, Lahni PM, Denenberg AG et al. Induction of endotoxin tolerance enhances bacterial clearance and survival in murine polymicrobial sepsis. Shock 2008; 30:267-73.

9 Brinkmann V, Reichard U, Goosmann C et al. Neutrophil extracellular traps kill bacteria. Science 2004; 303:1532-5.

10 Institute of Laboratory Animal Resources (US). Committee on Care and Use of Laboratory Animals., National Institutes of Health (US). Division of Research Resources. Guide for the care and use of laboratory animals. Rev. 1985 edn. Bethesda, MD: US Department of Health and Human Services, Public Health Service, National Insititutes of Health, 1985.

11 Biewenga J, van der Ende MB, Krist LF, Borst A, Ghufron M, van Rooijen N. Macrophage depletion in the rat after intraperitoneal administration of liposome-encapsulated clodronate: depletion kinetics and accelerated repopulation of peritoneal and omental macrophages by administration of Freund's adjuvant. Cell Tissue Res 1995; 280:189-96.

12 van Rooijen N, Hendrikx E. Liposomes for specific depletion of macrophages from organs and tissues. Methods Mol Biol 2010; 605:189-203.

13 van Rooijen N, van Kesteren-Hendrikx E. 'In vivo' depletion of macrophages by liposome-mediated 'suicide'. Methods Enzymol 2003; 373:3-16.

14 Mulligan MS, Jones ML, Bolanowski MA et al. Inhibition of lung inflammatory reactions in rats by an anti-human IL-8 antibody. J Immunol 1993; 150:5585-95.

15 Schierloh P, Yokobori N, Aleman M et al. Mycobacterium tuberculosis-induced gamma interferon production by natural killer cells requires cross talk with antigen-presenting cells involving Toll-like receptors 2 and 4 and the mannose receptor in tuberculous pleurisy. Infect Immun 2007; 75:5325-37.

16 Fuchs TA, Abed U, Goosmann C et al. Novel cell death program leads to neutrophil extracellular traps. J Cell Biol 2007; 176:23141.

17 Klonz A, Wonigeit K, Pabst R, Westermann J. The marginal blood pool of the rat contains not only granulocytes, but also lymphocytes, NK-cells and monocytes: a second intravascular compartment, its cellular composition, adhesion molecule expression and interaction with the peripheral blood pool. Scand J Immunol 1996; 44:461-9.

18 Fernandez GC, Lopez MF, Gomez SA et al. Relevance of neutrophils in the murine model of haemolytic uraemic syndrome: mechanisms involved in Shiga toxin type 2-induced neutrophilia. Clin Exp Immunol 2006; 146:76-84.

19 Betsuyaku T, Liu F, Senior RM et al. A functional granulocyte colony-stimulating factor receptor is required for normal chemoattractant-induced neutrophil activation. J Clin Invest 1999; 103:825-32.

20 Anderson BO, Brown JM, Shanley PF, Bensard DD, Harken AH.
Marginating neutrophils are reversibly adherent to normal lung endothelium. Surgery 1991; 109:51-61.

21 Doerschuk CM, Allard MF, Martin BA, MacKenzie A, Autor AP, Hogg JC. Marginated pool of neutrophils in rabbit lungs. J Appl Physiol 1987; 63:1806-15.

22 Peters AM, Saverymuttu SH, Bell RN, Lavender JP. Quantification of the distribution of the marginating granulocyte pool in man. Scand J Haematol 1985; 34:111-20.

23 Allen L, Weatherford T. Role of fenestrated basement membrane in lymphatic absorption from peritoneal cavity. Am J Physiol 1959; 197:551-4.

24 Murphey ED, Fang G, Sherwood ER. Endotoxin pretreatment improves bacterial clearance and decreases mortality in mice challenged with Staphylococcus aureus. Shock 2008; 29:512-8.

25 Murphey ED, Fang G, Varma TK, Sherwood ER. Improved bacterial clearance and decreased mortality can be induced by LPS tolerance and is not dependent upon IFN-gamma. Shock 2007; 27:289-95.

26 Erroi A, Fantuzzi G, Mengozzi M et al. Differential regulation of cytokine production in lipopolysaccharide tolerance in mice. Infect Immun 1993; 61:4356-9.

27 Lepe-Zuniga JL, Klostergaard J. Tolerance to endotoxin in vitro: independent regulation of interleukin-1, tumor necrosis factor and interferon alpha production during in vitro differentiation of human monocytes. Lymphokine Res 1990; 9:309-19.

28 Seatter SC, Li MH, Bubrick MP, West MA. Endotoxin pretreatment of human monocytes alters subsequent endotoxin-triggered release of inflammatory mediators. Shock 1995; 3:252-8.

29 Murphey ED, Fang G, Sherwood ER. Pretreatment with the Grampositive bacterial cell wall molecule peptidoglycan improves bacterial clearance and decreases inflammation and mortality in mice challenged with Staphylococcus aureus. Crit Care Med 2008; 36:3067-73.

30 Benjamim CF, Ferreira SH, Cunha FQ. Role of nitric oxide in the failure of neutrophil migration in sepsis. J Infect Dis 2000; 182:214-23.

31 Matute-Bello G, Frevert CW, Kajikawa O et al. Septic shock and acute lung injury in rabbits with peritonitis: failure of the neutrophil response to localized infection. Am J Respir Crit Care Med 2001; 163:234-43.

32 Alves-Filho JC, de Freitas A, Spiller F, Souto FO, Cunha FQ. The role of neutrophils in severe sepsis. Shock 2008; 30 (Suppl. 1):3-9.

33 Abraham E, Arcaroli J, Shenkar R. Activation of extracellular signal-regulated kinases, NF-kappa B, and cyclic adenosine $5^{\prime}$-monophosphate response element-binding protein in lung neutrophils occurs by differing mechanisms after hemorrhage or endotoxemia. J Immunol 2001; 166:522-30.

34 Shenkar R, Abraham E. Mechanisms of lung neutrophil activation after hemorrhage or endotoxemia: roles of reactive oxygen intermediates, NF-kappa B, and cyclic AMP response element binding protein. J Immunol 1999; 163:954-62.

35 Coldren CD, Nick JA, Poch KR et al. Functional and genomic changes induced by alveolar transmigration in human neutrophils. Am J Physiol Lung Cell Mol Physiol 2006; 291:L1267-76.

36 Williams JH, Jr, Moser KM, Ulich T, Cairo MS. Harvesting the noncirculating pool of polymorphonuclear leukocytes in rats by hetastarch exchange transfusion (HET): yield and functional assessment. J Leukoc Biol 1987; 42:455-62.

37 Ermert D, Urban CF, Laube B, Goosmann C, Zychlinsky A, 
Brinkmann V. Mouse neutrophil extracellular traps in microbial infections. J Innate Immun 2009; 1:181-93.

38 Clark SR, Ma AC, Tavener SA et al. Platelet TLR4 activates neutrophil extracellular traps to ensnare bacteria in septic blood. Nat Med 2007; 13:463-9.

39 Zinkernagel AS, Timmer AM, Pence MA et al. The IL- 8 protease SpyCEP/ScpC of group A Streptococcus promotes resistance to neutrophil killing. Cell Host Microbe 2008; 4:170-8.

$40 \mathrm{Lu} \mathrm{M}$, Varley AW, Ohta S, Hardwick J, Munford RS. Host inactivation of bacterial lipopolysaccharide prevents prolonged tolerance following Gram-negative bacterial infection. Cell Host Microbe 2008; 4:293-302.

41 Rearte B, Maglioco A, Balboa L et al. Mifepristone (RU486) restores humoral and $\mathrm{T}$ cell-mediated immune response in endotoxin immunosuppressed mice. Clin Exp Immunol 2010; 162:568-77.

42 Parker LC, Jones EC, Prince LR, Dower SK, Whyte MK, Sabroe I. Endotoxin tolerance induces selective alterations in neutrophil function. J Leukoc Biol 2005; 78:1301-5. 